\title{
Review of Traffic Sign Detection and Recognition Techniques
}

\author{
Swati Dehariya \\ M.Tech Scholar \\ Department of Electronics and Communication Engineering \\ All Saint College of Technology, Bhopal, India
}

\author{
Prof. M. Sarwar Raeen \\ Professor \& HOD \\ Department of Electronics and Communication Engineering \\ All Saint College of Technology, Bhopal, India
}

\begin{abstract}
Text, as one of the most compelling developments of humankind, has assumed a significant job in human life, so distant from antiquated occasions. The rich and exact data epitomized in content is extremely helpful in a wide scope of vision-based applications; along these lines content detection and recognition in regular scenes have turned out to be significant and dynamic research points in PC vision and report investigation. Traffic sign detection and recognition is a field of connected PC vision research worried about the programmed detection and grouping or recognition of traffic signs in scene pictures procured from a moving vehicle. Driving is an assignment dependent on visual data handling. The traffic signs characterize a visual language translated by drivers. Traffic signs convey much data important for effective driving; they portray current traffic circumstance, characterize option to proceed, preclude or grant certain headings. In this paper, talked about different detection and recognition schemes.
\end{abstract}

Keywords- Traffic, Text, Detection, Recognition, Image.

$* * * * *$

\section{INTRODUCTION}

Over the most recent three decades there was an expansion of street traffic, despite the fact that the quantity of individuals executed or genuinely harmed in street mishaps has diminished. This demonstrates regardless of whether our streets are currently more packed than any other time in recent memory, they are more secure due the fundamental advances in vehicle design, for example, improved fold zones and side effect bars. This can likewise be assigned by aloof innovation, similar to safety belts, airbags, and electronically monitored slowing mechanisms. As indicated by the office for vehicle.

\section{A. Difficulties in detecting and recognizing traffic signs} From the outset locate the goal of TSDR is very much characterized and is by all accounts very straightforward. Lets consider a camera that is mounted into a vehicle. This camera catches a surge of pictures and the framework identifies and perceives the traffic signs in the recovered pictures. Sadly there are, other than the positive angles, likewise some negative viewpoints.

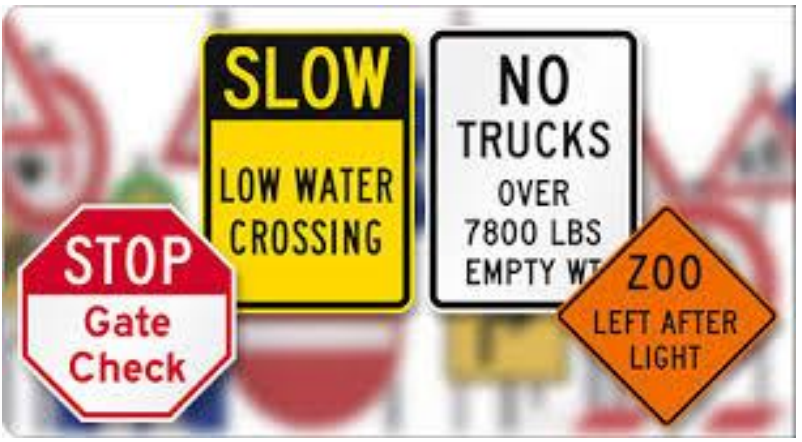

Figure 1: traffic Signs
The positive parts of TSDR is the uniqueness of the design of traffic signs, hues differentiate typically very well against the earth, the signs are carefully situated in respect to the earth and are frequently set up in an unmistakable sight to the driver.

Then again, there are as yet various negative parts of TSDR. We can recognize the accompanying viewpoints:

-Lightning conditions are variable and not controllable. Lightning is distinctive as per the time and season, climate conditions and nearby light varieties, for example, heading of light.

-The nearness of different articles like people on foot, trees, different vehicles, boards, and structures. This can cause fractional impediment and shadows. The items or encompassing could be like traffic signs by shading or shape.

-The sign establishment and surface material can physically change after some time, affected by mishaps and climate, along these lines bringing about muddled and harmed signs and deteriorated hues.

- The recovered pictures from the camera of a moving vehicle regularly experiences movement obscure and vehicle vibration.

-It is preposterous to expect to produce a disconnected model of all the potential appearances of the sign, on the grounds that there are such a significant number of degrees of opportunity. The item size relies upon the separation to the camera. Moreover, the camera isn't constantly opposite to the signs, which creates a perspective change. 
- The detection and recognition of traffic signs are made up for lost time with the exhibition of a framework continuously. This requires a framework with proficient calculations and ground-breaking equipment.

-Traffic signs exists in several variations regularly unique in relation to legitimately characterized guidelines.

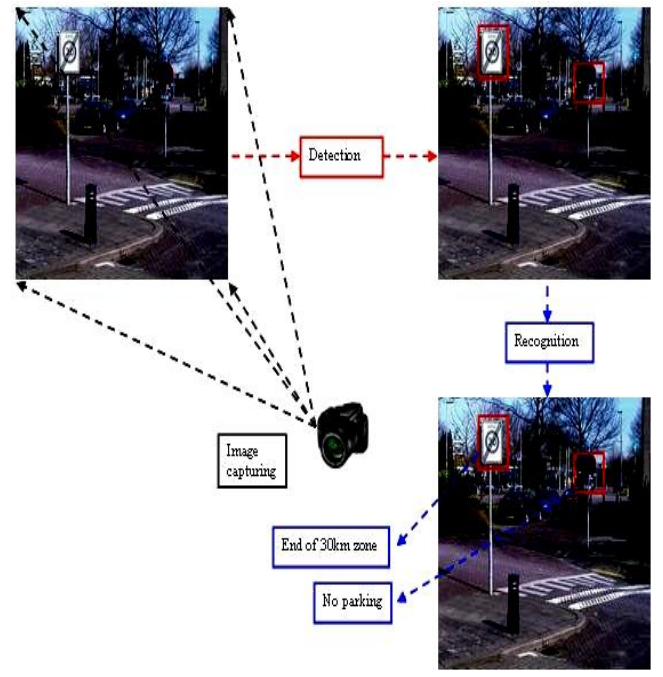

Figure 2: Simple overview of the traffic sign recognition system

Thus, to construct a successful TSDR system one must provide a large number of traffic sign examples to make the system respond correctly to real traffic images. This requires large databases what is expensive and a time consuming task.

\section{LITERATURE SURVEY}

Y. Zhu et al.,[1] In this work, it is propose a novel content based traffic sign detection structure with two profound learning parts. All the more absolutely, it is apply a completely convolutional system to portion applicant traffic sign zones giving up-and-comer districts of intrigue (return on initial capital investment), trailed by a quick neural system to recognize messages on the extricated return on initial capital investment. The proposed strategy utilizes the qualities of traffic signs to improve the effectiveness and precision of content detection. On one hand, the proposed two-organize detection strategy diminishes the inquiry zone of content detection and expels messages outside traffic signs. Then again, it takes care of the issue of multi-scales for the content detection part to an enormous degree. Broad trial results demonstrate that the proposed technique accomplishes the cutting edge results on the freely accessible traffic sign informational collection: Traffic Guide Board informational collection. Also, it is gather an informational index of content based traffic signs including Chinese and English traffic signs. Our technique likewise performs well on this informational collection, which exhibits that the proposed strategy is general in distinguishing traffic signs of various dialects.

M. Javanmardi et al.,[2] Detection of traffic signs and light posts utilizing light detection and running (LiDAR) information has shown a substantial commitment to street wellbeing enhancements. In this investigation, the creators propose a quick and dependable strategy, which can recognize different traffic signs and light shafts in portable LiDAR information. In particular, they first utilize the surface remaking calculation to separate the typical vectors of the focuses as one of the trademark includes and apply $\mathrm{k}$ - implies on the trademark highlights of the focuses to consequently fragment the information into street or nonstreet focuses.

D. Wang et al.,[3] Programmed traffic sign detection is trying because of the multifaceted nature of scene pictures, and quick detection is required in genuine applications, for example, driver help frameworks. In this work, it is propose a quick traffic sign detection strategy dependent on a course technique with saliency test and neighboring scale mindfulness. In the course strategy, include maps of a few channels are separated proficiently utilizing estimate strategies. Sliding windows are pruned progressively utilizing coarse-to-fine classifiers and the relationship between's neighboring scales. The course framework has just one free parameter, while the various limits are chosen by an information driven methodology. To further expand speed, it is likewise utilize a novel saliency test dependent on mid-level highlights to pre-prune foundation windows.

T. Chen et al.,[4] Continuous traffic sign detection and recognition has been getting progressively more consideration lately because of the prevalence of driverhelp frameworks and self-sufficient vehicles. This work proposes a precise and productive traffic sign detection method by investigating AdaBoost and bolster vector relapse (SVR) for discriminative indicator learning. Not the same as the revealed traffic sign detection procedures, a novel saliency estimation approach is first proposed, where another saliency model is fabricated dependent on the traffic sign-explicit shading, shape, and spatial data. By consolidating the saliency data, improved element pyramids are worked to gain proficiency with an AdaBoost model that recognizes a lot of traffic sign applicants from pictures.

Á. González et al.,[5] Traffic sign detection and recognition has been completely contemplated for quite a while. In any case, traffic board detection recognition still remains a test in $\mathrm{PC}$ vision because of its various kinds and the immense fluctuation of the data portrayed in them. This 
work displays a strategy to identify traffic boards in road level pictures and to perceive the data contained on them, as an application to insightful transportation frameworks (ITS). The fundamental reason can be to make a programmed stock of the traffic boards situated in a street to help street support and to help drivers.

Table 1: Summery of Literature Review

\begin{tabular}{|c|c|c|c|}
\hline $\begin{array}{l}\text { Sr } \\
\text { No. }\end{array}$ & $\begin{array}{l}\text { Author } \\
\text { Name \& } \\
\text { Year }\end{array}$ & $\begin{array}{l}\text { Proposed } \\
\text { Work }\end{array}$ & Outcome \\
\hline 1 & $\begin{array}{l}\text { Y. } \quad \text { Zhu, } \\
\text { IEEE } 2018\end{array}$ & $\begin{array}{l}\text { Novel text- } \\
\text { based traffic } \\
\text { sign detection }\end{array}$ & $\begin{array}{lr}\text { Precision } & 0.90 \\
\text { Recall } & 0.87 \\
\text { F-measure } & 0.88\end{array}$ \\
\hline 2 & $\begin{array}{l}\text { M. } \\
\text { Javanmardi, } \\
\text { IEEE } 2018\end{array}$ & $\begin{array}{l}\text { Apply k- } \\
\text { means on the } \\
\text { characteristic } \\
\text { features }\end{array}$ & $\begin{array}{l}\text { Robustness of the } \\
\text { proposed method }\end{array}$ \\
\hline 3 & $\begin{array}{l}\text { D. Wang, } \\
\text { IEEE } 2017\end{array}$ & $\begin{array}{l}\text { Proposed } \\
\text { cascade } \\
\text { method }\end{array}$ & Fast calculation \\
\hline 4 & $\begin{array}{l}\text { T. Chen, } \\
\text { IEEE } 2016\end{array}$ & $\begin{array}{l}\text { Proposed } \\
\text { support vector } \\
\text { regression }\end{array}$ & $\begin{array}{l}\text { Robust and obtains } \\
\text { superior accuracy } \\
\text { and efficiency. }\end{array}$ \\
\hline 5 & $\begin{array}{l}\text { Á. } \\
\text { González, } \\
\text { IEEE } 2014\end{array}$ & $\begin{array}{l}\text { Applying blue } \\
\text { and white } \\
\text { color } \\
\text { segmentation }\end{array}$ & $\begin{array}{l}\text { Real images from } \\
\text { Google Street } \\
\text { View prove the } \\
\text { efficiency }\end{array}$ \\
\hline
\end{tabular}

\section{TRAFFIC SIGN DETECTION AND RECOGNITION SYSTEM}

The ID of traffic signs is generally practiced in two fundamental stages: detection and recognition. In the detection stage we can recognize the accompanying parts: pre-handling, include extraction, and division. As should be obvious an entire chain of picture handling steps are required to at long last recognize the traffic signs. The initial phase in the detection stage is pre-preparing, which may incorporate a few activities. These tasks amends a picture which is impacted by clamor, movement obscure, out-of-center haze, bending brought about by low goals, etcetera. Besides, highlight pictures are removed from the first picture. These element pictures containing significant data of the first picture, yet in a diminished portrayal. From there on, the traffic signs must be isolated from the foundation. Implying that districts of steady highlights and discontinuities must be recognized by division. This should be possible with basic division methods and with the more modern division procedures. After the division stage pursues another component extraction part, yet this time dependent on abnormal state picture investigation. In the last piece of the detection stage are the potential traffic signs identified from the fragmented pictures, by utilizing the removed highlights of the past part. The productivity and speed of the detection stage are significant factors in the entire procedure, since it lessens the hunt space and shows just potential areas. After detection we can further examine the picture with a few activities and alter it or concentrate further important data of it.

Detection and Recognition can be done by following steps and Approaches-

- Pre-processing.

- Feature extraction.

- Segmentation.

- Detection.

- Classification and recognition.

Approaches-

- Neural Network

- Optical Character Recognition

- Maximally Stable External Regions

Table 2: Techniques used by various TSR system

\begin{tabular}{|c|c|c|}
\hline Stages & & Methods \\
\hline Preprocessing & \multicolumn{2}{|c|}{ Camera calibration and its resolution } \\
\hline \multirow{6}{*}{ Detection } & \multirow{2}{*}{$\begin{array}{l}\text { Color } \\
\text { based }\end{array}$} & Color Thresholding \\
\hline & & Color Learning \\
\hline & & Edge Detection \\
\hline & Shape & Template Algorithm \\
\hline & & $\begin{array}{l}\text { Machine learning } \\
\text { techniques }\end{array}$ \\
\hline & & Hough Transform \\
\hline Recognition & & late Matching \\
\hline & & hine Learning \\
\hline
\end{tabular}

\section{A. STEPS}

i. Pre-Processing

The goal of pre-processing is to adjust an image so that the resulting image is more suitable than the original. An image pre-processing method that works for one application may not work very well for another application. The input of the pre-processing part consist of the original (sensor) image and the output is a reconstructed, restored, and enhanced image. The input can be influenced by noise, motion blur, out-of-focus blur, distortion caused by low resolution, etcetera. We can split the image pre-processing methods in two different domains:

- Spatial domain operates directly on the pixels. 
- Frequency domain operates on the Fourier transform of an image.

\section{ii. Feature extraction}

If the input to an algorithm is too large to be processed or there is much data without much useful information, then the input will be transformed into a reduced representation set of features. This transformation is called feature extraction. Its objective is to select the right set of features which describes the data in a sufficient way without loss of accuracy. The set of all possible features represents a feature space

\section{iii. Segmentation}

Segmentation refers to operations that partitions an image into regions that are consistent with respect to some conditions. The goal of segmentation is to simplify or change the representation of an image into something that is more meaningful or easier to analyze. The basic attribute for colour segmentation is image luminance amplitude for a monochrome image and colour components for a colour image. Image shape and texture are also useful attributes for segmentation.

\section{iv. Detection}

The segmentation part provide us with potential regions of traffic signs. The goal of the detection part is the identification of these potential regions with the use of rules that accept or reject a potential region as a traffic sign candidate. There also exist two different approaches in the traffic sign detection part: colour based and shape based. Based on the segmentation results, shape analysis is in general applied to these results in order to perform the detection of the traffic signs.

\section{v. Recognition phase}

The output of the detection phase is a list of detected traffic sign. This list is forwarded to the recognition phase for further evaluation. To design a good recognizer, many features should be taken into account. Firstly, the recognizer should present a good discriminative power and low computational cost. Secondly, it should be robust to the geometrical status of the sign, such as the vertical or horizontal orientation, the size, and the position of the sign in the image. Thirdly, it should be robust 5to noise. Fourthly, the recognition should be carried out quickly if it is designed for real time applications. Furthermore, the recognizer must be able to learn a large number of classes and as much as possible a priori knowledge about traffic signs should be employed into the classifier design.

\section{B. APPROACHES}

i. Neural Network

Transform the original image into the gray scale image by using support vector machines, then use convolutional neural networks with fixed and learnable layers for detection and recognition. The fixed layer can reduce the amount of interest areas to detect, and crop the boundaries very close to the borders of traffic signs. The learnable layers can increase the accuracy of detection significantly. Besides, we use bootstrap methods to improve the accuracy and avoid overfitting problem

\section{ii. Optical Character Recognition}

Optical character recognition or optical character reader (OCR) is the mechanical or electronic conversion of images of typed, handwritten or printed text into machineencoded text, whether from a scanned document, a photo of a document, a scene-photo.

\section{iii. Maximally Stable External Regions}

After detecting the shape of the traffic signs, optical character recognition (OCR) method is used to recognize the character present in it. A technique based on Maximally Stable External Regions (MSER) region and canny edge detector was also supervised for character recognition in traffic sign detection process.

\section{CONCLUSION}

This paper gives an overview of three, widely used, techniques on the topic of traffic sign detection and recognition. Statistical methods seem limited in this field and therefore much research has been done to find methods that are more accurate. As a final word, the choice of a method and the use of a technique depend on the complexity of the problem specific task. It can be a time consuming job to find the right settings of the different techniques. The study of the three emphasized methods in traffic sign detection and recognition can be easily extended with more research. Therefore we can combine techniques and achieve significant better results.

\section{REFERENCE}

[1]. Y. Zhu, M. Liao, M. Yang and W. Liu, "Cascaded Segmentation-Detection Networks for Text-Based Traffic Sign Detection," in IEEE Transactions on Intelligent Transportation Systems, vol. 19, no. 1, pp. 209-219, Jan. 2018.

[2]. M. Javanmardi, Z. Song and X. Qi, "Automated traffic sign and light pole detection in mobile LiDAR scanning data," in IET Intelligent Transport Systems, vol. 13, no. 5, pp. 803-815, 52018

[3]. D. Wang, X. Hou, J. Xu, S. Yue and C. Liu, "Traffic Sign Detection Using a Cascade Method With Fast Feature Extraction and Saliency Test," in IEEE Transactions on 
Intelligent Transportation Systems, vol. 18, no. 12, pp. 3290-3302, Dec. 2017

[4]. T. Chen and S. Lu, "Accurate and Efficient Traffic Sign Detection Using Discriminative AdaBoost and Support Vector Regression," in IEEE Transactions on Vehicular Technology, vol. 65, no. 6, pp. 4006-4015, June 2016.

[5]. Á. González, L. M. Bergasa and J. J. Yebes, "Text Detection and Recognition on Traffic Panels From StreetLevel Imagery Using Visual Appearance," in IEEE Transactions on Intelligent Transportation Systems, vol. 15, no. 1, pp. 228-238, Feb. 2014.

[6]. Y. Yuan, Z. Xiong and Q. Wang, "An Incremental Framework for Video-Based Traffic Sign Detection, Tracking, and Recognition," in IEEE Transactions on Intelligent Transportation Systems, vol. 18, no. 7, pp. 1918-1929, July 2017.

[7]. Q. Hu, S. Paisitkriangkrai, C. Shen, A. van den Hengel and F. Porikli, "Fast Detection of Multiple Objects in Traffic Scenes With a Common Detection Framework," in IEEE Transactions on Intelligent Transportation Systems, vol. 17, no. 4, pp. 1002-1014, April 2016.

[8]. T. Chen and S. Lu, "Accurate and Efficient Traffic Sign Detection Using Discriminative AdaBoost and Support Vector Regression," in IEEE Transactions on Vehicular Technology, vol. 65, no. 6, pp. 4006-4015, June 2016.

[9]. X. Yuan, J. Guo, X. Hao and H. Chen, "Traffic Sign Detection via Graph-Based Ranking and Segmentation Algorithms," in IEEE Transactions on Systems, Man, and Cybernetics: Systems, vol. 45, no. 12, pp. 1509-1521, Dec. 2015.

[10].Á. González, L. M. Bergasa and J. J. Yebes, "Text Detection and Recognition on Traffic Panels From StreetLevel Imagery Using Visual Appearance," in IEEE Transactions on Intelligent Transportation Systems, vol. 15, no. 1, pp. 228-238, Feb. 2014.

[11].A. Mammeri, A. Boukerche and M. Almulla, "Design of traffic sign detection, recognition, and transmission systems for smart vehicles," in IEEE Wireless Communications, vol. 20, no. 6, pp. 36-43, December 2013.

[12].J. Greenhalgh and M. Mirmehdi, "Real-Time Detection and Recognition of Road Traffic Signs," in IEEE Transactions on Intelligent Transportation Systems, vol. 13, no. 4, pp. 1498-1506, Dec. 2012 